\title{
In utero hypoxia altered Ang II-induced contraction via PKC $\beta$ in fetal cerebral arteries
}

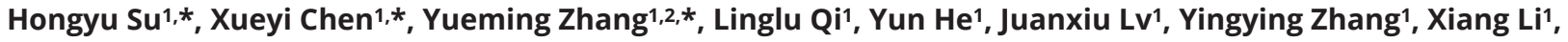 \\ Jiaqi Tang1 and Zhice $\mathbf{X u}^{1}$ \\ ${ }^{1}$ Institute for Fetology, First Hospital of Soochow University, Suzhou, Jiangsu, China \\ 2Department of Obstetrics and Gynecology, First Hospital of Soochow University, Suzhou, Jiangsu, China
}

Correspondence should be addressed to J Tang or Z Xu: tangjiaqi75@163.com or xuzhice@suda.edu.cn

${ }^{*}(\mathrm{H}$ Su, $\mathrm{X}$ Chen and $\mathrm{Y}$ Zhang contributed equally to this work)

\begin{abstract}
Cerebral circulation is important in fetal brain development, and angiotensin II (Ang II) plays vital roles in regulation of adult cerebral circulation. However, functions of Ang II in fetal cerebral vasculature and influences of in utero hypoxia on Ang II-mediated fetal cerebral vascular responses are largely unknown. This study investigated the effects and mechanisms of in utero hypoxia on fetal middle cerebral arteries (MCA) via Ang II. Near-term ovine fetuses were exposed to in utero hypoxia, and fetal MCA responses to Ang II were tested for vascular tension, calcium transient, and molecular analysis. Ang II caused significant dose-dependent contraction in control fetal MCA. Ang II-induced MCA constriction was decreased significantly in hypoxic fetuses. Neither losartan (AT1R antagonist, 10-5 mol/L) nor PD123,319 (AT2R antagonist, 10-5 mol/L) altered Ang II-mediated contraction in fetal MCA. Phenylephrine-mediated constriction was also significantly weaker in hypoxic fetuses. Bay K8644 caused similar contractions between the two groups. Protein expression of L-type voltage-dependent calcium channels was unchanged. There were no differences in caffeine-mediated vascular tension or calcium transients. Contraction induced by PDBu (PKC agonist) was obviously weaker in hypoxic MCA. Protein expression of $\mathrm{PKC} \beta$ was reduced in the hypoxic compared with the control, along with no differences in phosphorylation levels. The results showed that fetal MCA was functionally responsive to Ang II near term. Intrauterine hypoxia reduced the vascular agonist-mediated contraction in fetal MCA, probably via decreasing PKC $\beta$ and its phosphorylation, which might play protective effects on fetal cerebral circulation against transient hypoxia.
\end{abstract}

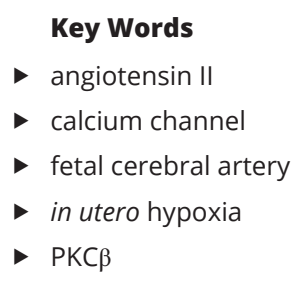

- calcium channel

fetal cerebral artery

- $\mathrm{PKC} \beta$

\section{Introduction}

Neonatal encephalopathy affects approximately 3 in 1000 births, inducing perinatal brain injury (Hagberg et al. 2016). It is well known that perinatal brain injury is commonly due to hypoxia-ischemia during pregnancy and delivery (Kurinczuk et al. 2010). Although central circulation is important in protecting the fetal brain from hypoxia, there is very limited information on Ang II-mediated fetal cerebral circulation exposed to perinatal hypoxia-ischemia.

The mammalian fetus is highly adapted to a relatively low level of physiological hypoxia in utero. 
Diseases and adverse environments during pregnancy such as severe preeclampsia, placenta previa, umbilical cord compression, and exposure to high altitude (Gao et al. 2018), can cause pathological intrauterine hypoxia. Previous studies demonstrated that high-altitude hypoxia increased cerebrovascular morbidity, by altering potassium chloride, endothelin-1, serotonin-induced contractile responses (Longo et al. 1993, Silpanisong et al. 2017) and nitric oxide-soluble guanyly cyclase-cyclic guanosine monophosphate-protein kinase G (NO-sGC-cGMP-PKG) pathway-elicited vasodilation in fetal cerebral arteries (Williams \& Pearce 2006, Thorpe et al. 2017). Angiotensin II (Ang II) and the renin-angiotensin system (RAS) exist in the brain locally and play important roles in regulating cerebral circulation in adults. However, it is unknown if the RAS in fetal brain vascular systems is sufficiently mature to elicit responses and if Ang II is also crucial in regulation of fetal cerebral arteries. In addition, whether in utero hypoxia can alter Ang II-mediated vascular functions in fetal cerebral arteries is unknown too.

Chronic moderate intrauterine hypoxia can cause fetal growth restriction and decrease vascular density (Castillo-Melendez et al. 2015, 2017). Partial compression of the umbilical cord produces fetal hypertension and reduces cardiac output and blood flow to hepatic, renal, musculoskeletal, and pulmonary vascular beds (Iwamoto et al. 1991, Gardner et al. 2001). Severe umbilical cord occlusion in the near-term ovine fetus results in a rapid decrease in the availability of oxygen to the brain (Kaneko et al. 2003). Intermittent umbilical cord occlusion may contribute to adverse neurodevelopment, including alterations in synapse formation and neuronal circuitry (Nishigori et al. 2008, Czikk et al. 2015). However, influences and mechanisms of intrauterine hypoxia on Ang II-induced constriction in fetal cerebral arteries are still largely unknown.

Angiotensin II is an important vasoconstrictor agent upon binding its receptors, AT1R and AT2R. In adult cerebral arteries, AT1R mainly mediates vascular constriction, while AT2R may mediate vasodilation (Tang et al. 2017). Following combining with its $G$ proteincoupled receptors, a series of calcium channels and calciumsensitive signaling pathway are triggered to regulate vascular tone. L-type voltage-dependent calcium channels and ryanodine receptor-operated calcium channels are predominant in the regulation of vascular tone. Protein kinase C (PKC) can induce calcium-sensitive signaling pathways, participating in regulating vascular tension, and contributing to abnormal contraction in disease (El-Yazbi et al. 2015). Chronic hypoxia or high-altitude hypoxia can sustain changes in PKC-dependent pathways (Goyal et al. 2010, Silpanisong et al. 2017). PDBu, a PKC agonist, can phosphorylate ERK1/2 so as to regulate $\mathrm{Ca}^{2+}$ sensitivity (Zhao et al. 2003, Goyal et al. 2009).

This study determined direct actions of Ang II and its receptor subtypes on fetal MCA. We also explored the effects and mechanisms of in utero hypoxia on fetal cerebral arteries, especially via calcium channels and PKC signaling pathways.

\section{Materials and methods}

\section{Animals}

All procedures used were approved by the Ethical Committee of the First Hospital of Soochow University and the Institutional Animal Care and Use Committee.

\section{Preparation, surgery, and postoperative care}

Pregnant sheep (Hu sheep, purchased from Zhejiang sheep company, $n=22$ ) were surgically instrumented using strict aseptic techniques at gestational day $122 \pm 3$ (term: approximately 145 days). In brief, food and water were with-held for $24 \mathrm{~h}$ prior to surgery. Anesthesia was induced by intramuscular injection of atropine and ketamine. Then the ewe was placed on her back on surgical table followed by trachea intubation with the aid of a laryngoscope. Operative anesthesia was maintained by spontaneous inhalation of $1.5-2.0 \%$ isoflurane in $\mathrm{O}_{2}$. The abdomen and flanks were shaved and scrubbed with $75 \%$ ethanol three times and another spray of concentrated iodine solution. The ewe was covered with three layers of sterile surgical linen cloths. The midline incision site was exposed and midline abdominal incisions, and uterine incisions were then made, the fetal hind limbs were exteriorized, minimizing the loss of amniotic fluid. Catheters were inserted into fetal femoral arteries on both sides. Then, an inflatable occluder was placed around the umbilical cord. Fetal skin incisions were closed with thin silk suture and the uterine incision was closed in layers. The occluder and catheters were then exteriorized via an incision and were packaged in a bag sticking to the flank. At this time the anesthetic was turned off and the ewe was ventilated until spontaneous respiration occurred. The ewe was allowed to recover in a cage with free access to food and water.

Sprague-Dawley male rats were killed at 5 month old $(n=10)$ to harvest their middle cerebral artery (MCA), coronary artery (COA), and mesenteric artery (MA). 
Pregnant rats at gestational day $21(n=5)$ were subjected to cesarean surgeries to obtain the fetus, and fetal aorta was gently isolated from the surrounding tissues.

\section{Intrauterine hypoxia and arterial gas}

After 5-7 days of surgical recovery, the compression of the umbilical cord was performed to reduce umbilical blood flow until fetal arterial oxygen pressure decreased to about $50 \%$ of the baseline. The compression was maintained for $30 \mathrm{~min}$, and then the occluder was deflated, allowing return of umbilical blood flow to baseline. This hypoxia treatment was repeated every day for a total of 5 days. The fetus exposed to intrauterine hypoxia was defined as the hypoxic group. Its twin fetus without intrauterine hypoxia served as the control. At baseline $(0.5 \mathrm{~h}$ prior to hypoxia treatment) and $1 \mathrm{~min}$ following intrauterine hypoxia, fetal arterial blood samples were collected immediately for determination of arterial oxygen pressure and other blood values using a blood gas analyzer (Gem Premier 3000).

\section{Measurements of vascular tension}

After 5 days of intermittent intrauterine hypoxia, ewes were killed for collection of fetal MCA. Fetal cerebral arteries were isolated immediately from the connective tissue and cut into small rings. Vascular rings were mounted gently in an $M$ series Myograph chamber (Radnoti Glass Technology, Covina, CA, USA) filled with HEPES-PSS solution (mmol/L: $\mathrm{NaCl} 141.85, \mathrm{KCl} 4.7$, $\mathrm{MgSO}_{4}$ 1.7, EDTA 0.51, $\mathrm{CaCl}_{2} \cdot 2 \mathrm{H}_{2} \mathrm{O} 2.79, \mathrm{KH}_{2} \mathrm{PO}_{4}$ 1.17, glucose 5.0 and HEPES 10.0, pH 7.4), using $40 \mu \mathrm{m}$ stainless steel wire. The tension was recorded with PowerLab and Labchart 7. Potassium chloride $(0.12 \mathrm{~mol} / \mathrm{L})$ was used to measure maximum contractile capability and served

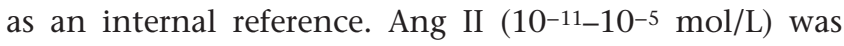
added in the presence or absence of losartan $\left(10^{-5} \mathrm{~mol} / \mathrm{L}\right)$ or PD123,319 (10-5 mol/L) (Xiao et al. 2008, Wu et al. 2014). Phenylephrine (PhE, 10-9-10-4 mol/L), Bay K8644

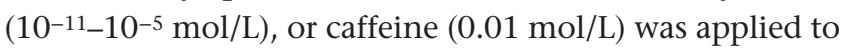
the MCA for measuring vascular tension. All the drugs were diluted with HEPES-PSS solution. Losartan's bioactivity was tested in fetal aorta, adult MCA, COA, and MA.

\section{Calcium transient in single smooth muscle cell}

Single smooth muscle cells from fetal MCA were isolated enzymatically (Tang et al. 2017) and adhered to the chamber glass. Then smooth muscle cells were loaded in $\mathrm{Ca}^{2+}$-free PSS solution with fura2-AM $(2 \mu \mathrm{mol} / \mathrm{L}$, Invitrogen) for $30 \mathrm{~min}$ at room temperature. The fluorescence was excited at $340 / 380 \mathrm{~nm}$ wavelength with IonOptix. The calcium transient was calculated qualitatively using the fluorescence ratio of fura2-AM at 340 and $380 \mathrm{~nm}$ (F340/F380). Caffeine (0.01 mol/L) was added to detect calcium transients via ryanodine receptoroperated calcium channels.

\section{Real time-PCR}

Total RNA was extracted from fetal cerebral arteries with RNAiso reagent (TaKaRa). The template RNA $(2 \mu \mathrm{g})$ was reverse transcribed with PrimeScript ${ }^{\mathrm{TM}}$ by using 1st-strand cDNA synthesis kits (TakaRa, Cat\#6210A). The reference sequence of the genes was acquired from the UCSC Genome Browser. Gene primer sequences are shown in Table 1. Q-PCR was performed on a Bio-Rad myiQ2 thermal cycler q-PCR machine with a SYBR Premix Ex Taq ${ }^{\mathrm{TM}}$ mix. Data were normalized against GAPDH as internal control and calibrated with normal control cDNA. The ratio of relative mRNA expression was calculated with the $2^{-\Delta \Delta \mathrm{Ct}}$ method.

\section{Western blot}

Fetal cerebral arteries were grinded to powder, and lysed in RIPA Lysis Buffer (Beyotime Biotechnology, Shanghai, China; Cat\#P0013B), containing 1\% Protease Inhibitor Cocktail (Bimake, Houston, TX, USA; Cat\#B14001) and 1\% phosphatase inhibitor cocktail A and B (Bimake, Cat\#B15001) for $30 \mathrm{~min}$. Then, the homogenates was centrifuged at $13,800 \mathrm{~g}$ for $30 \mathrm{~min}$, and the supernatant was collected. Proteins were separated using sodium dodecyl sulfate polyacrylamide gel electrophoresis (SDS-PAGE) and then transferred to polyvinylidene fluoride (PVDF) membranes (Immobilon-P Membrane, Cat\#IPUH00010). Membrane was incubated with primary antibodies at $4^{\circ} \mathrm{C}$ overnight, including GAPDH (Proteintech, Rosemont, IL, USA; Cat\#60004-1-1g, 1:10,000), AT1R (Proteintech, Cat\#25343-1-AP, Lot\#00022501, 1:1000), AT2R (Santa Cruz, Cat\#sc-9040, Lot\#G0910, 1:200), CACNA1C (Proteintech, Cat\#21774-1-AP, Lot\#00014709, 1:1000), PKC $\alpha$ (Cohesion Biosciences, London, UK; Cat\#CPA5525, Lot\#CP1D10A, 1:1000), PKC $\beta$ (Proteintech, Cat\#129191-AP, Lot\#00058186, 1:1000), phosphorylated-PKC $\beta$ at Ser660 (Cell Signaling Technology, Cat\#9371, Lot\#3, 1:1000) and Thr641 (Abcam, Cat\#ab194749, Lot\#GR31779814, 1:1000), according to the supplier's instructions. The corresponding secondary antibody was 
Table 1 Gene primer sequences.

\begin{tabular}{l}
\hline Gene name \\
\hline GAPDH \\
AT1R \\
AT2R \\
CACNA1C \\
CACNB1 \\
CACNB2 \\
CACNB3 \\
$P K C \alpha$ \\
$P K C \beta$ \\
$P K C \delta$ \\
$P K C \varepsilon$ \\
\hline
\end{tabular}

\begin{tabular}{l}
\hline Forward primer $\left(5^{\prime}-3^{\prime}\right)$ \\
\hline ATGGAAAGGCCATCACCATC \\
GTAATTGTGCCTTCCAGCTTTG \\
GGAAGTGCCAGGTCAATGAC \\
GGACACTGCTGTGGACCTTCA \\
TATTTGGACGTTGAGGTGTTGGA \\
TTGCATTTGCAGTTCGGACAA \\
TCCAACACTACTAGCTGCAGGGAT \\
CCTCATGTACCACATCCAGCAG \\
CAACCAAGACCTTCTGTGGCA \\
GCTGTGGTCCAGCAACAC \\
TATGAGCTCATGGCATCTCTTG
\end{tabular}

\begin{tabular}{l}
\hline Reverse primer $\left(5^{\prime}-3^{\prime}\right)$ \\
\hline CATCACAAACATGGGAGCGT \\
CCAGAAGGTCTGCATCCAGG \\
GCAACATGTTATTTCGGAATCAG \\
GATCTCTGTTGTGTCATTCAGGG \\
TTGGTTGCTCTGGACGCTGA \\
TTGGAATGAATCCGATTTCACAG \\
TCCTGGCAAGAGGACCATCAT \\
CCTTCCGAGTCCAGCATGAC \\
CGTTGTGTTCCATGATCGACT \\
CGACCTGATGTACCACATCCA \\
AGTTCATGGCCACCTACCTTC
\end{tabular}

incubated for $1 \mathrm{~h}$, and included a horseradish peroxidase (HRP)-conjugated goat anti-rabbit $\operatorname{IgG}(\mathrm{H}+\mathrm{L})$ antibody (Multisciences, Hangzhou, China; Cat\#GAR0072, Lot\#A90400133, 1:5000) or goat anti-mouse $\operatorname{IgG}(\mathrm{H}+\mathrm{L})$ antibody (Multisciences, Cat\#GAM0072, Lot\#A90390143, 1:5000). The bands were visualized using an imaging system (Tanon Science \& Technology Company, Shanghai, China). Imaging signals were digitized and analyzed, and then the ratio of interest protein to GAPDH was obtained to quantify the relative protein expression.

\section{Data analysis}

All data were presented as means \pm S.E.M. Dose response curves were analyzed using two-way ANOVA followed by Bonferroni post hoc tests. Averages were compared using $t$ test or Mann-Whitney test, where appropriate. For all comparisons, $P<0.05$ represented statistical significance.
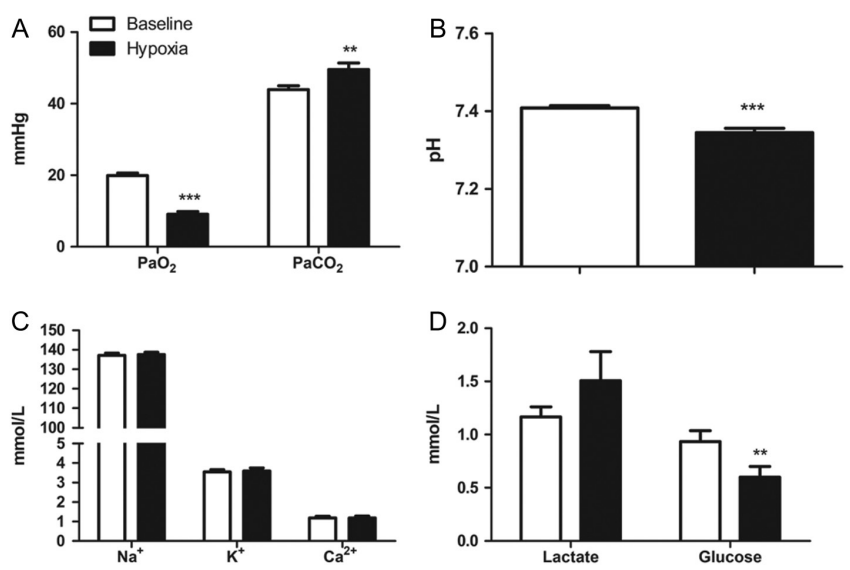

\section{Figure 1}

Arterial oxygen pressure, carbon dioxide pressure, and other indices in ovine fetuses. Figure 1 shows arterial oxygen pressure $\left(\mathrm{PaO}_{2}\right)$, carbon dioxide pressure $\left(\mathrm{PaCO}_{2}\right), \mathrm{pH}$ value, and concentrations of $\mathrm{Na}^{+}, \mathrm{K}^{+}, \mathrm{Ca}^{2+}$, lactate, and glucose in fetal blood at baseline and after intrauterine hypoxia (Hypoxia) at 1 min following hypoxia that lasted 30 min. ${ }^{*} P<0.05$; $\star \star P<0.01 ; * \star * P<0.001 . n=10-13$.

\section{Results}

\section{Fetal arterial oxygen pressure, carbon dioxide pressure, and other indexes}

Fetal arterial oxygen pressure was decreased immediately after compression of the umbilical cord $(19.92 \pm 2.53$ $\mathrm{mmHg}$ vs $9.08 \pm 2.72 \mathrm{mmHg}$ ) at $1 \mathrm{~min}$ following hypoxia (Fig. 1A). Carbon dioxide pressure was increased during intrauterine hypoxia (Fig. 1A). The $\mathrm{pH}$ value was decreased from $7.41 \pm 0.02$ to $7.34 \pm 0.04$ (Fig. 1B). No significant differences were found in the concentration of $\mathrm{Na}^{+}, \mathrm{K}^{+}$, $\mathrm{Ca}^{2+}$, or lactate (Fig. 1C and D). There was a reduction in glucose concentration $(\mathrm{mmol} / \mathrm{L})$ from $0.934 \pm 0.102$ to $0.601 \pm 0.099$ with intrauterine hypoxia (Fig. 1D).

\section{Angiotensin II-mediated vascular constriction and its receptors}

Contractile responses caused by $\mathrm{KCl}(0.12 \mathrm{~mol} / \mathrm{L})$ showed no significant differences between the two groups (Fig. $2 \mathrm{~A})$. In the MCA, when compared to the control, Ang II-induced constriction was weaker in the fetal hypoxia group (Fig. 2B and C).

Ang II mediates vascular constriction mainly via its receptors (AT1R or AT2R) in adult arteries. Losartan (a specific inhibitor of AT1R, 10-5 mol/L) or PD123,319 (a specific inhibitor of AT2R, $10^{-5} \mathrm{~mol} / \mathrm{L}$ ) was used. In ovine fetal MCA, the inhibitory effect of losartan on the Ang II-mediated contractile responses was not significant (Fig. $3 \mathrm{~A}$ and $\mathrm{B})$, which was markedly different from that in adults. Incubation with PD123,319 also did not change Ang II-induced constriction of the fetal MCA (Fig. 3C and D). The mRNA expression of AT1R was significantly lower in the fetal MCA exposed to hypoxia (Fig. 3E), but AT1R protein expression was not changed between the two groups (Fig. 3F). AT2R mRNA and protein expressions showed no significant differences between the two groups. 

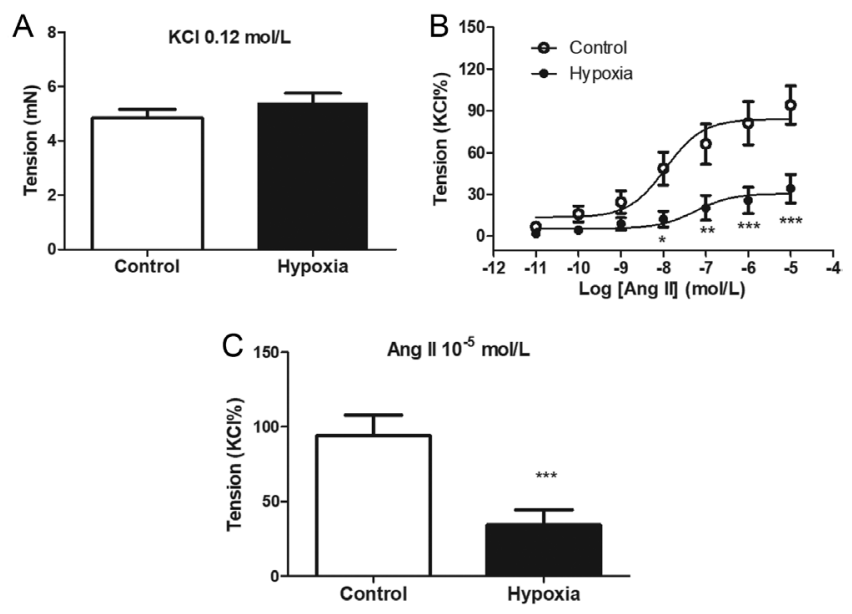

\section{Figure 2}

Angiotensin II-induced vasoconstriction in fetal middle cerebral arteries. Potassium chloride $(\mathrm{KCl}, 0.12 \mathrm{~mol} / \mathrm{L})$ and cumulative doses of angiotensin II (Ang II, ranging from 10-11 to $10^{-5} \mathrm{~mol} / \mathrm{L}$ ) mediated vascular constriction in the control and intrauterine hypoxic group (Hypoxia) (A and B). (C) Shows the comparison in Ang II (10-5 $\mathrm{mol} / \mathrm{L})$-mediated contraction between the control and intrauterine hypoxic group. $* P<0.05$; $* \star P<0.01$; $\star \star \star * P<0.001 . n=10-15$

Figure 3G and $\mathrm{H}$ demonstrated losartan's bioactivity at $10^{-5} \mathrm{~mol} / \mathrm{L}$ in adult rat MCA, MA, and COA, and in fetal rat aorta.

\section{Functions of calcium channels in fetal MCA}

In addition to Ang II, PhE was applied to determine fetal vascular tone (Fig. 4A). Significantly decreased constriction caused by PhE was similar to that by Ang II in the hypoxic group, indicating that the common downstream pathways might be changed by hypoxia. To determine what caused the weaker contraction in the fetal MCA exposed to intrauterine hypoxia, L-type voltagedependent calcium channels and ryanodine receptoroperated calcium channels were tested. Bay K8644, an activator of L-type voltage-dependent calcium channels, mediated similar contractile responses in the two groups (Fig. 4B). Despite decreased mRNA expression of CACNA1C following intrauterine hypoxia, no remarkable difference was found in its protein levels (Fig. 4C and D), suggesting that the functions of L-type calcium channels and their downstream pathways were unchanged in response to intrauterine hypoxia. Ryanodine receptor-operated calcium channels were then tested using caffeine. Caffeine induced very similar vascular tension in fetal MCA as well as the same calcium transient in the isolated MCA smooth muscle cells in the two groups (Fig. 3E and F). These data demonstrated that neither L-type calcium channels nor ryanodine receptor-operated calcium channels were functionally altered by intermittent in utero hypoxia.

\section{Protein kinase $\mathrm{C}$ in regulating MCA tone}

Cumulative doses of $\mathrm{PDBu}$ (PKC agonist) caused constriction of fetal MCA, and the constriction was weaker in the intrauterine hypoxic group than in the control (Fig. 5A and B), similar to the responses to Ang II and PhE. The mRNA expression of PKC $\alpha$ and $\beta$ was decreased in the hypoxic group, while PKC $\delta$ and $\varepsilon$ mRNA levels were unchanged (Fig. 5C). PKC $\alpha$ protein expression showed no notable differences between the two groups (Fig. 5D and E). PKC $\beta$ protein expression was remarkably decreased in the fetal MCA exposed to intrauterine hypoxia (Fig. $5 \mathrm{D}$ and $\mathrm{E}$ ), whereas the ratio of $\mathrm{PKC} \beta$ phosphorylation at Ser660 and Thr641 to total PKC $\beta$ was not changed by intrauterine hypoxia (Fig. 5D, E and F). These results suggested that the decreased PKC $\beta$ with its concomitant reduced phosphorylation/activation might mediate weaker vascular constriction in the fetal MCA exposed to intrauterine hypoxia.

\section{Discussion}

The present study demonstrated two important findings. The first finding showed that Ang II regulated nearterm fetal MCA, the key artery supplying blood and oxygen to the brain, suggesting that ovine fetal MCA was functionally mature near term in response to Ang II. The second observation indicated that in utero hypoxia (approximately 50\% reduction in fetal arterial oxygen pressure) decreased vascular contractions, presumably via PKC $\beta$ in fetal cerebral arteries.

It is well known that central Ang II plays important roles in the control of adult MCA tone (Tang et al. 2017). However, it is unknown whether during early development Ang II regulates fetal MCA. This study presented repeatable data that Ang II reliably produced significant constriction of near-term ovine fetal MCA. The MCA maximal response to Ang II was $84.04 \%$ of that induced by $\mathrm{KCl}$ in functional experiments, demonstrating a relatively strong vasoconstriction. This is the first study to show that Ang II-mediated regulation of fetal cerebral vasculature is functionally developed near term. The clinical significance of this finding includes that many babies face hypoxia risks during perinatal periods and delivery, which has been proved to be a major cause of neonatal encephalopathy (Li et al. 2017). Since the MCA 
A

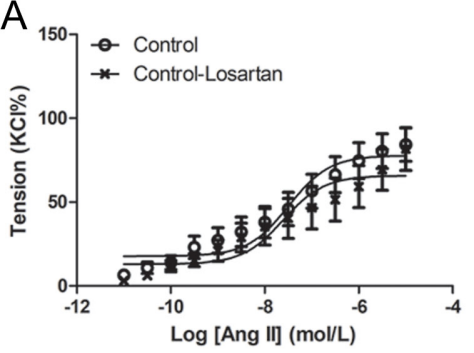

C
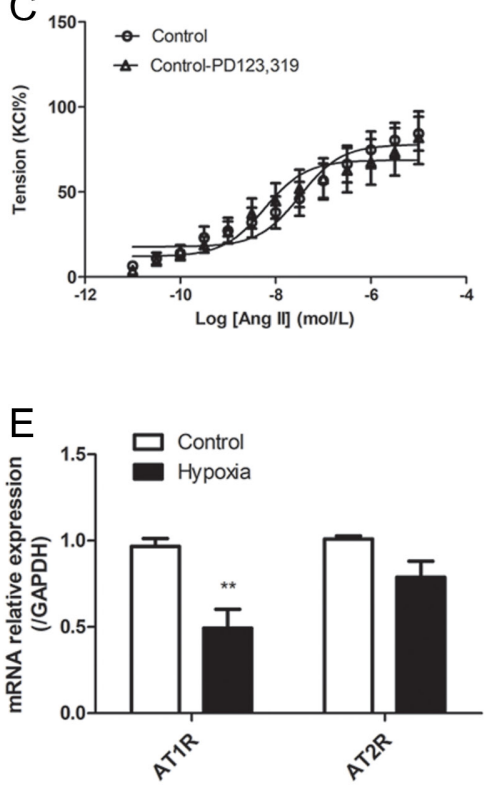

$\mathrm{G}$ Losartan in fetal and adult artery

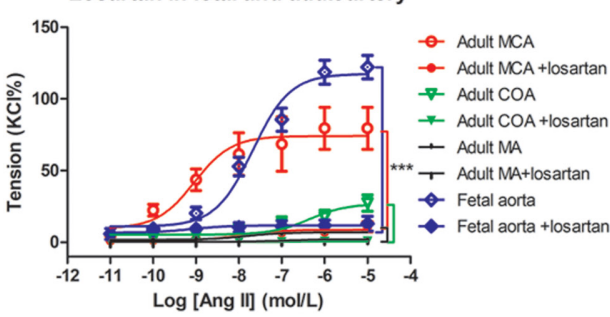

B

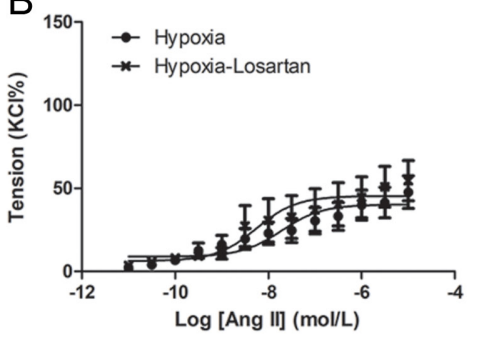

D
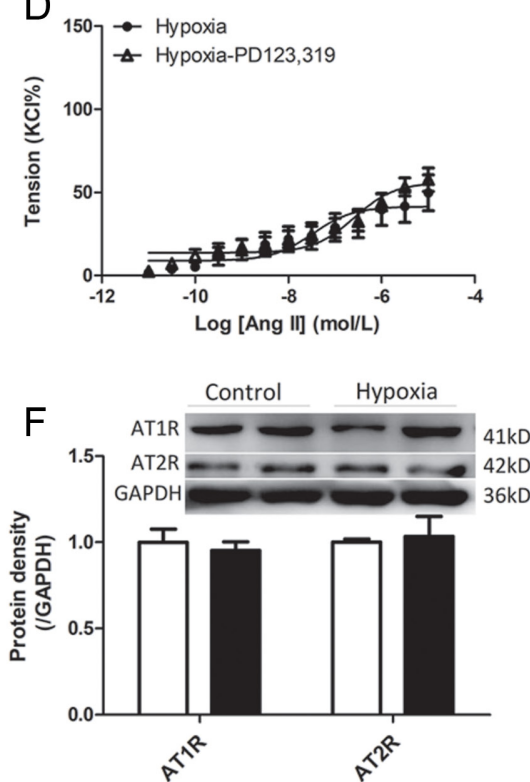

$\mathrm{H}$

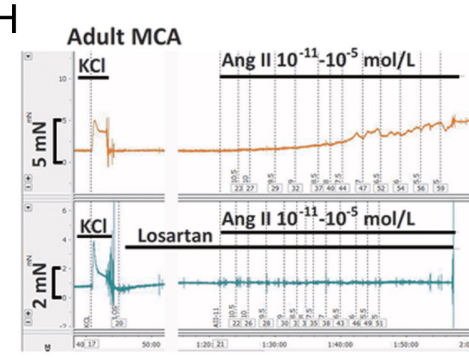

Figure 3

Functions of AT1 R and AT2R in fetal middle cerebral arteries. (A, B, C and D) Presents the vasoconstriction induced by cumulative doses of angiotensin II (Ang II) with or without losartan (AT1R inhibitor, 10-5 $\mathrm{mol} / \mathrm{L}$ ) or PD123,319 (AT2R inhibitor, $10^{-5} \mathrm{~mol} / \mathrm{L}$ ) in fetal middle cerebral arteries. (E and F) Presents the mRNA and protein expression of AT1R and AT2R. ( $G$ and H) Shows the detection of losartan's bioactivity in adult rat arteries (MCA, mesenteric artery, and coronary artery) and in fetal aorta from pregnant rat, and the image record in adult MCA with or without losartan. ${ }^{* *} P<0.01 ; * \star \star P<0.001$. Pregnant sheep: $n=12-15$. is a key artery in the brain to supply blood and nutrients to the central nervous system, the regulation of MCA is certainly important for protecting the brain from hypoxia.

Previous studies in other experimental models demonstrated that hypoxia or other environmental factors can affect the RAS in cardiovascular systems (Gao et al. 2017, Liu et al. 2018). Chronic hypoxia by exposure to high altitudes during pregnancy can potentiate fetal cerebrovascular constriction caused by endothelin-1 (Silpanisong et al. 2017). However, the influence of in utero hypoxia on fetal cerebral arteries in response to Ang II was unknown, while the RAS plays important roles in controlling cerebral arteries in the adult brain (Paulson et al. 1988, Pires et al. 2013). This study was the first to show that in utero hypoxia could affect Ang II-mediated vascular regulation of Ang II in fetal cerebral circulation. Hypoxia redistributes and changes blood flow to the fetal brain (Richardson et al. 1996), and the present study demonstrated that the decreased constrictions of fetal cerebral arteries might reduce vascular resistance, contributing to re-establishing the blood supply to the brain.

The questions were then raised, what were the mechanisms underlying the Ang II-produced vasoconstrictions of the fetal MCA? Was the effect in the fetal MCA the same as that in the adult MCA? Addressing these questions is important for fetal and newborn central physiology and pathophysiology under conditions of 

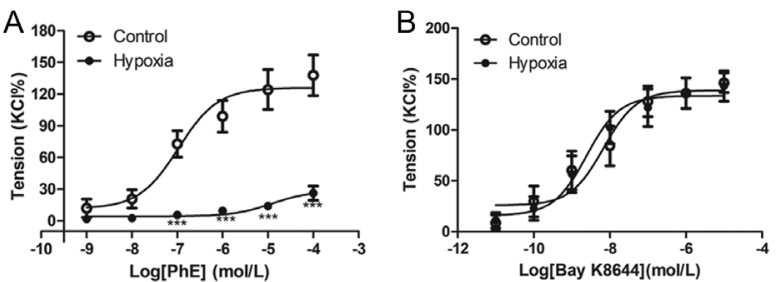

C
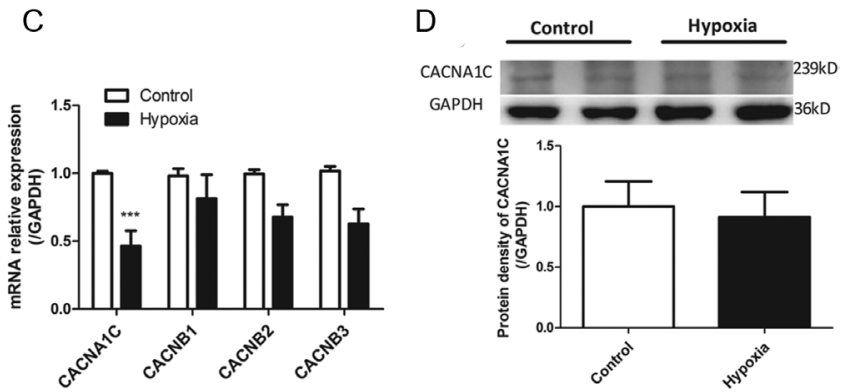

$\mathrm{E}$

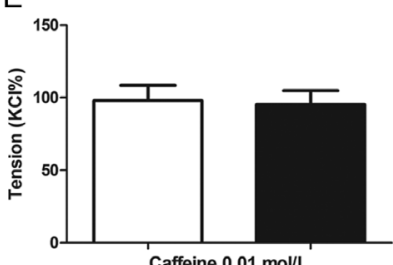

$\mathrm{F}$

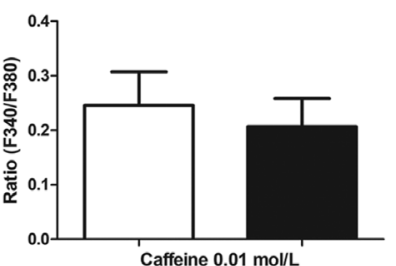

Figure 4

Involvement of L-type voltage-dependent calcium channels and ryanodine receptor-operated calcium channels in regulating fetal middle cerebral arteries. (A) Shows doses-response curves of phenylephrine (PhE) induced constriction in both groups. (B) Shows Bay K8644 (L-type calcium channel agonist)-mediated constriction in fetal cerebral arteries. (C and D) Presents the mRNA and protein expression of L-type voltage-dependent calcium channels. ( $E$ and $F)$ Shows caffeine $(0.01 \mathrm{~mol} / \mathrm{L}$, ryanodine receptor-operated calcium channel agonist)-induced tension in vessels and calcium transients in single smooth muscle cells. $\star \star \star P<0.001 . n=9-13$.

hypoxia. Thus, we tested two subtypes of Ang II receptors that have been proven functionally critical for cerebral vascular systems in adults. Notably, there had been no work to demonstrate Ang II receptors in fetal cerebral arteries, and this study firstly found expression of both mRNA and protein of the AT1R and AT2R in fetal MCA, providing new evidence of a local RAS in fetal central arteries during development. However, it was surprising that the specific AT1 antagonist losartan at a relatively high dose $\left(10^{-5} \mathrm{~mol} / \mathrm{L}\right)$ did not significantly block Ang II-generated vasoconstriction as expected in ovine fetal cerebral arteries, although losartan $\left(10^{-5} \mathrm{~mol} / \mathrm{L}\right)$ was able to diminish Ang II-mediated constriction in almost all peripheral arteries as well as adult MCA (Xiao et al. 2008, Tang et al. 2017). Thus, we carefully verified the bioactivity of the antagonist losartan and the equipment used with the same drug, same preparations, and same systems. The results proved that losartan $\left(10^{-5} \mathrm{~mol} / \mathrm{L}\right)$ could efficiently block Ang II-stimulated vasoconstriction of adult rat MCA, coronary arteries, and mesenteric arteries, demonstrating that there were no technical problems related to the antagonist (losartan) and systems used for testing. Those results also suggested that the fetal vascular system might act very differently from that of adults. The same drug was tested on fetal arteries, and the results showed that losartan $\left(10^{-5} \mathrm{~mol} / \mathrm{L}\right)$ also significantly inhibited Ang II-produced vasoconstriction of the fetal aorta, indicating that the effects of Ang II on fetal peripheral arteries were mainly via AT1R receptors. Finally, we increased the number of fetal MCA from the original $n=8$ to $n=20$ to avoid possible variations commonly seen in functional experiments. The results still showed that losartan did not significantly suppress Ang II-mediated constriction of the fetal MCA. Moreover, the AT2R blocker did not show any inhibitory effects in the fetal MCA, which was similar to the response of the adult model (Henrion et al. 2001, Xiao et al. 2008). Molecular testing demonstrated that mRNA expression of AT1R was lower in fetal MCA exposed to hypoxia, despite no differences in protein expression.
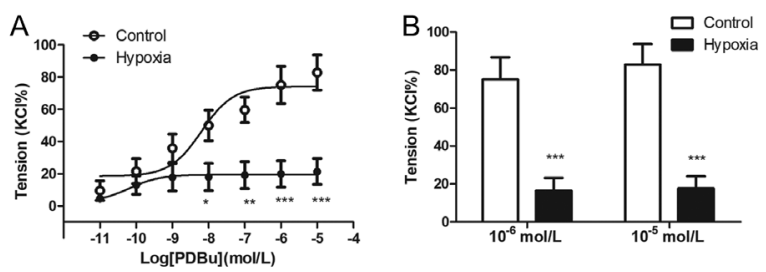

C
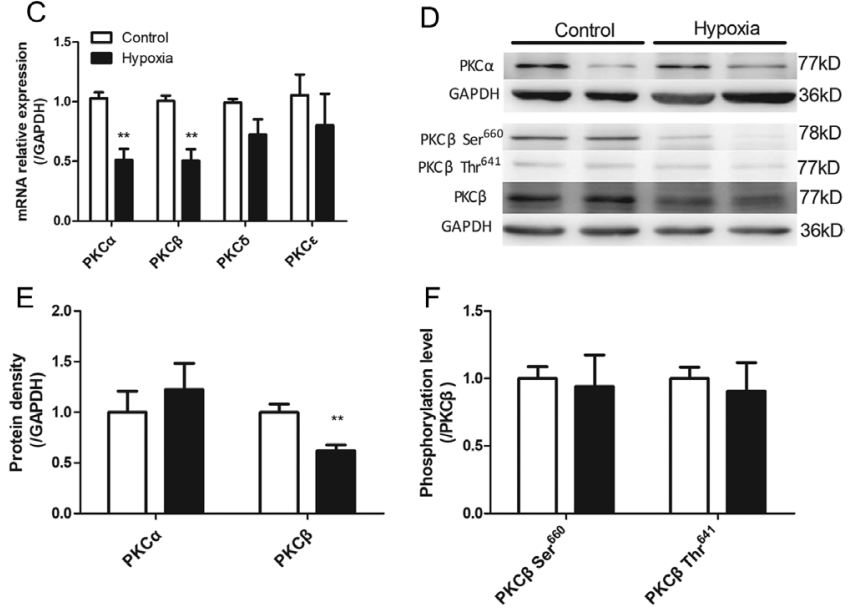

\section{Figure 5}

Involvement of protein kinase $\mathrm{C}$ in the regulation of fetal middle cerebral arteries. (A and B) Presents PDBu (PKC agonist)-mediated contractile responses in fetal middle cerebral arteries and the comparison of PDBu $\left(10^{-6} \mathrm{~mol} / \mathrm{L}, 10^{-5} \mathrm{~mol} / \mathrm{L}\right)$-mediated constriction of vessels from hypoxic versus normoxic groups. (C) Shows the mRNA expression of PKC subtypes. (D, E and F) Presents the protein expressions of $P K C \alpha$, and $\beta$, and the phosphorylation levels at Ser660 and Thr641 sites. ${ }^{*} P<0.05$; $\star * P<0.01 ; * * * P<0.001 . n=15$. 
AT2R mRNA and protein expression was unchanged between the two groups. Together, neither losartan nor PD123,319 blocked Ang II-mediated MCA constrictions in the fetus, making an explanation complicated. Shi et al. reported that intracerebroventricular high-dose losartan potentiated systemic blood pressure and Ang II-induced mean arterial pressure in vivo in near-term ovine fetuses (Shi et al. 2005), indicating that losartan might act differently in the fetal brain. Although Ang II receptors are divided into four subtypes, in general, only AT1R and AT2R, particularly AT1R, have been considered major types involved in the regulation of vascular systems (Montezano et al. 2014), while functional effects of AT3R and AT4R are unclear, even in adult models. The present study demonstrated that neither AT1R nor AT2R subtype blockers at $10^{-5} \mathrm{~mol} / \mathrm{L}$ could inhibit Ang II-stimulated fetal MCA constrictions, indicating that there might be other pathways for Ang II's actions in the fetal MCA. Whether these might involve AT3R or AT4R or other routes is definitely worth further investigation.

The next question was whether vascular constriction stimulated by other vascular agonists, for instance, PhE, would be reduced in the hypoxic group. The results showed similar weaker responses in fetal MCA induced by $\mathrm{PhE}$ in the hypoxic group, suggesting there might be common mechanisms. Vascular responses mainly depend on calcium channels and PKC pathways (Ringvold \& Khalil 2017). Our subsequent experiments showed that Bay K8644 mediated similar constriction in the two groups. Caffeine-mediated vascular tension at tissue level and calcium transients in isolated smooth muscle cells at the cell level showed no significant differences between the two groups. These data demonstrated that L-type voltage-dependent calcium channels and ryanodine receptor-operated calcium channels were not markedly altered by in utero hypoxia, and they were not the primary reason leading to the weaker MCA constriction observed in hypoxic fetuses.

In addition to calcium channels, PKC participates in the regulation of vascular tone. Protein kinases $\mathrm{C}$ can cause constrictions by several mechanisms, including regulation of ion channels, $\mathrm{Ca}^{2+}$ sensitization of contractile proteins, and other contractile pathways (Ringvold \& Khalil 2017). However, in some instances, PKC also can mediate dilation (Xu et al. 2019). In the present study, cumulative doses of PDBu (a PKC agonist) caused constriction in fetal MCA. When compared with the control, PDBu-induced fetal vascular constriction was attenuated significantly in the hypoxic group, accompanied by reduced mRNA and protein expression of PKC $\beta$. Meanwhile, the ratio of phosphorylation at $\mathrm{Ser}^{660}$ and $\mathrm{Thr}^{641}$ to total PKC $\beta$ showed no significant differences between the two groups. These findings provide new information to suggest that altered PKC $\beta$ levels may play a critical role in intrauterine hypoxia-induced weaker constriction in response to Ang II and PhE in the fetal MCA.

Hypoxia and ischemia can induce translocation of PKC isoforms (Takeishi et al. 1999, Pourmahram et al. 2008). The role of PKC $\beta$, either pathogenic or protective in fetal MCA, has not been determined. Previous work reported that a PKC $\beta$ inhibitor (ruboxistaurin) attenuated ischemia-reperfusion injury (Chen et al. 2014), and РКC $\beta$ null mice were protected against ischemia-reperfusion injury in the lung (Fujita et al. 2004). Gene knockdown of PKC 32 via small interfering RNA inhibits p66 (Shc) overexpression and phosphorylation in Caco-2 cells exposed to hypoxia-reperfusion (Chen et al. 2014). Glycine and dicoscin alleviate hypoxia-reperfusion injury via inhibiting РКC $\beta$ over-expression in the gastrointestinal system and heart (Liu et al. 2015, Hu et al. 2016, Zhang et al. 2018). PKC inhibitors Ro318220 and Go6976 significantly suppress peroxide (for instance, $\mathrm{H}_{2} \mathrm{O}_{2}$ )elicited constriction of pulmonary arteries (Pourmahram et al. 2008). These results that indicated РКС $\beta$ inactivation might have protective effects against ischemia-reperfusion injury (Zhang et al. 2018). The present study showed that when the fetus was exposed to intrauterine hypoxia, PKC $\beta$ was down-regulated in fetal cerebral arteries, which might have protective functions against excessive constriction and ensure adequate blood flow to the brain. It is the first work proving that in utero hypoxia alleviated Ang II-induced constriction, in association with altered PKC $\beta$. Thus, PKC $\beta$ inhibitors might benefit fetal circulation and prevent perinatal brain injury caused by hypoxia-ischemia. Detailed mechanism studies should be performed to determine how fetal hypoxia alters PKC $\beta$ levels. The signaling pathway involving Ang II and PKC $\beta$ in the fetal MCA was affected by in utero hypoxia.

In conclusion, we found that Ang II played critical roles in regulating fetal MCA, controlling central blood flow near term. In utero hypoxia reduced Ang II- and PhEmediated vascular constriction of fetal cerebral arteries, which might moderate blood flow to the fetal brain under conditions of hypoxia. Interestingly, neither losartan nor PD123,319 at relatively high doses $\left(10^{-5} \mathrm{~mol} / \mathrm{L}\right)$ altered Ang II-induced contraction in the fetal MCA, which was quite different from adult MCA, and worth further investigation. L-type voltage-dependent calcium channels and ryanodine receptor-operated calcium channels were not altered by in utero hypoxia in fetal cerebral vasculature. 
However, decreased PKC $\beta$ was closely associated with hypoxia-reduced fetal MCA constriction, which leads to a new hypothesis that PKC $\beta$ downregulation might act as a protective mechanism to maintain cerebral blood flow and protect against in utero hypoxia-induced perinatal brain injury.

\section{Declaration of interest}

The authors declare that there is no conflict of interest that could be perceived as prejudicing the impartiality of the research reported.

\section{Funding}

Supported by NSFC (81771592, 81370714, and 81320108006), Talent Fund of Famous City and Hospital (3101030421), and Jiangsu Key Discipline/ Laboratory (Fetology) Fund.

\section{Acknowledgements}

Jiaqi Tang and Zhice Xu performed the surgery and constructed the in utero hypoxia model. Hongyu Su, Xueyi Chen, and Yueming Zhang accomplished the vascular experiments. Linglu Qi and Yun He performed the molecular detection. Juanxiu Lv provided guidance on anesthetic methods. Yingying Zhang and Xiang Li offered help in maintaining the sheep and sampling collections. Jiaqi Tang wrote the manuscript. Zhice $\mathrm{Xu}$ revised the manuscript.

\section{References}

Castillo-Melendez M, Yawno T, Allison BJ, Jenkin G, Wallace EM \& Miller SL 2015 Cerebrovascular adaptations to chronic hypoxia in the growth restricted lamb. International Journal of Developmental Neuroscience 45 55-65. (https://doi.org/10.1016/j. ijdevneu.2015.01.004)

Castillo-Melendez M, Yawno T, Sutherland A, Jenkin G, Wallace EM \& Miller SL 2017 Effects of antenatal melatonin treatment on the cerebral vasculature in an ovine model of fetal growth restriction. Developmental Neuroscience 39 323-337. (https://doi. org/10.1159/000471797)

Chen Z, Wang G, Zhai X, Hu Y, Gao D, Ma L, Yao J \& Tian X 2014 Selective inhibition of protein kinase $\mathrm{C}$ beta 2 attenuates the adaptor P66 Shc-mediated intestinal ischemia-reperfusion injury. Cell Death and Disease 5 e1164. (https://doi.org/10.1038/cddis.2014.131)

Czikk MJ, Totten S, Hammond R \& Richardson BS 2015 Microtubuleassociated protein 2 and synaptophysin in the preterm and near-term ovine fetal brain and the effect of intermittent umbilical cord occlusion. Reproductive Sciences 22 367-376. (https://doi. org/10.1177/1933719114529371)

El-Yazbi AF, Abd-Elrahman KS \& Moreno-Dominguez A 2015 PKCmediated cerebral vasoconstriction: role of myosin light chain phosphorylation versus actin cytoskeleton reorganization. Biochemical Pharmacology 95 263-278. (https://doi.org/10.1016/j.bcp.2015.04.011)

Fujita T, Asai T, Andrassy M, Stern DM, Pinsky DJ, Zou YS, Okada M, Naka Y, Schmidt AM \& Yan SF 2004 PKCbeta regulates ischemia/ reperfusion injury in the lung. Journal of Clinical Investigation 113 1615-1623. (https://doi.org/10.1172/JCI19225)
Gao Q, Tang J, Li N, Zhou X, Li Y, Liu Y, Wu J, Yang Y, Shi R, He A, et al. 2017 A novel mechanism of angiotensin II-regulated placental vascular tone in the development of hypertension in preeclampsia. Oncotarget 8 30734-30741. (https://doi.org/10.18632/ oncotarget.15416)

Gao Q, Tang J, Li N, Liu B, Zhang M, Sun M \& Xu Z 2018 What is precise pathophysiology in development of hypertension in pregnancy? Precision medicine requires precise physiology and pathophysiology. Drug Discovery Today 23 286-299. (https://doi.org/10.1016/j. drudis.2017.10.021)

Gardner DS, Fletcher AJ, Fowden AL \& Giussani DA 2001 A novel method for controlled and reversible long term compression of the umbilical cord in fetal sheep. Journal of Physiology 535 217-229. (https://doi. org/10.1111/j.1469-7793.2001.00217.x)

Goyal R, Mittal A, Chu N, Shi L, Zhang L \& Longo LD 2009 Maturation and the role of PKC-mediated contractility in ovine cerebral arteries. American Journal of Physiology: Heart and Circulatory Physiology 297 H2242-H2252. (https://doi.org/10.1152/ajpheart.00681.2009)

Goyal R, Mittal A, Chu N, Arthur RA, Zhang L \& Longo LD 2010 Maturation and long-term hypoxia-induced acclimatization responses in PKC-mediated signaling pathways in ovine cerebral arterial contractility. American Journal of Physiology: Regulatory, Integrative and Comparative Physiology 299 R1377-R1386. (https://doi.org/10.1152/ ajpregu.00344.2010)

Hagberg H, David Edwards A \& Groenendaal F 2016 Perinatal brain damage: the term infant. Neurobiology of Disease 92 102-112. (https:// doi.org/10.1016/j.nbd.2015.09.011)

Henrion D, Kubis N \& Levy BI 2001 Physiological and pathophysiological functions of the AT(2) subtype receptor of angiotensin II: from large arteries to the microcirculation. Hypertension 38 1150-1157. (https:// doi.org/10.1161/hy1101.096109)

Hu Y, Tao X, Han X, Xu L, Yin L, Qi Y, Zhao Y, Xu Y, Wang C \& Peng J 2016 Dioscin attenuates gastric ischemia/reperfusion injury through the down-regulation of PKC/ERK1/2 signaling via PKCalpha and PKCbeta2 inhibition. Chemico-Biological Interactions 258 234-244. (https://doi.org/10.1016/j.cbi.2016.09.014)

Iwamoto HS, Stucky E \& Roman CM 1991 Effect of graded umbilical cord compression in fetal sheep at 0.6-0.7 gestation. American Journal of Physiology 261 H1268-H1274. (https://doi.org/10.1152/ ajpheart.1991.261.4.H1268)

Kaneko M, White S, Homan J \& Richardson B 2003 Cerebral blood flow and metabolism in relation to electrocortical activity with severe umbilical cord occlusion in the near-term ovine fetus. American Journal of Obstetrics and Gynecology 188 961-972. (https://doi. $\operatorname{org} / 10.1067 / \mathrm{mob} .2003 .219)$

Kurinczuk JJ, White-Koning M \& Badawi N 2010 Epidemiology of neonatal encephalopathy and hypoxic-ischaemic encephalopathy. Early Human Development 86 329-338. (https://doi.org/10.1016/j. earlhumdev.2010.05.010)

Li B, Concepcion K, Meng X \& Zhang L 2017 Brain-immune interactions in perinatal hypoxic-ischemic brain injury. Progress in Neurobiology 159 50-68. (https://doi.org/10.1016/j.pneurobio.2017.10.006)

Liu Y, Jin J, Qiao S, Lei S, Liao S, Ge ZD, Li H, Wong GT, Irwin MG \& Xia Z 2015 Inhibition of PKCbeta2 overexpression ameliorates myocardial ischaemia/reperfusion injury in diabetic rats via restoring caveolin-3/Akt signaling. Clinical Science 129 331-344. (https://doi. org/10.1042/CS20140789)

Liu Y, Qi L, Wu J, Xu T, Yang C, Chen X, Lv J \& Xu Z 2018 Prenatal high-salt diet impaired vasodilatation with reprogrammed reninangiotensin system in offspring rats. Journal of Hypertension 36 2369-2379. (https://doi.org/10.1097/HJH.0000000000001865)

Longo LD, Hull AD, Long DM \& Pearce WJ 1993 Cerebrovascular adaptations to high-altitude hypoxemia in fetal and adult sheep. American Journal of Physiology 264 R65-R72. (https://doi.org/10.1152/ ajpregu.1993.264.1.R65) https://joe.bioscientifica.com

https://doi.org/10.1530/JOE-19-0370 (c) 2020 Society for Endocrinology Published by Bioscientifica Ltd. Printed in Great Britain 
Montezano AC, Nguyen Dinh Cat A, Rios FJ \& Touyz RM 2014 Angiotensin II and vascular injury. Current Hypertension Reports 16 431. (https://doi.org/10.1007/s11906-014-0431-2)

Nishigori H, Mazzuca DM, Nygard KL, Han VK \& Richardson BS 2008 BDNF and TrkB in the preterm and near-term ovine fetal brain and the effect of intermittent umbilical cord occlusion. Reproductive Sciences 15 895-905. (https://doi.org/10.1177/1933719108324135)

Paulson OB, Waldemar G, Andersen AR, Barry DI, Pedersen EV, Schmidt JF \& Vorstrup S 1988 Role of angiotensin in autoregulation of cerebral blood flow. Circulation 77 I55-I58.

Pires PW, Dams Ramos CM, Matin N \& Dorrance AM 2013 The effects of hypertension on the cerebral circulation. American Journal of Physiology: Heart and Circulatory Physiology 304 H1598-H1614. (https://doi.org/10.1152/ajpheart.00490.2012)

Pourmahram GE, Snetkov VA, Shaifta Y, Drndarski S, Knock GA, Aaronson PI \& Ward JP 2008 Constriction of pulmonary artery by peroxide: role of $\mathrm{Ca} 2+$ release and PKC. Free Radical Biology and Medicine 45 1468-1476. (https://doi.org/10.1016/j. freeradbiomed.2008.08.020)

Richardson BS, Carmichael L, Homan J, Johnston L \& Gagnon R 1996 Fetal cerebral, circulatory, and metabolic responses during heart rate decelerations with umbilical cord compression. American Journal of Obstetrics and Gynecology 175 929-936. (https://doi.org/10.1016/ s0002-9378(96)80027-5)

Ringvold HC \& Khalil RA 2017 Protein kinase C as regulator of vascular smooth muscle function and potential target in vascular disorders. Advances in Pharmacology 78 203-301. (https://doi.org/10.1016/ bs.apha.2016.06.002)

Shi L, Mao C, Thornton SN, Sun W, Wu J, Yao J \& Xu Z 2005 Effects of intracerebroventricular losartan on angiotensin II-mediated pressor responses and c-fos expression in near-term ovine fetus. Journal of Comparative Neurology 493 571-579. (https://doi.org/10.1002/cne.20802)

Silpanisong J, Kim D, Williams JM, Adeoye OO, Thorpe RB \& Pearce WJ 2017 Chronic hypoxia alters fetal cerebrovascular responses to endothelin-1. American Journal of Physiology: Cell Physiology $\mathbf{3 1 3}$ C207-C218. (https://doi.org/10.1152/ajpcell.00241.2016)

Takeishi Y, Jalili T, Ball NA \& Walsh RA 1999 Responses of cardiac protein kinase $\mathrm{C}$ isoforms to distinct pathological stimuli are differentially regulated. Circulation Research 85 264-271. (https://doi. org/10.1161/01.res.85.3.264)

Tang J, Li N, Chen X, Gao Q, Zhou X, Zhang Y, Liu B, Sun M \& Xu Z 2017 Prenatal hypoxia induced dysfunction in cerebral arteries of offspring rats. Journal of the American Heart Association 6 e006630. (https://doi. org/10.1161/JAHA.117.006630)

Thorpe RB, Hubbell MC, Silpanisong J, Williams JM \& Pearce WJ 2017 Chronic hypoxia attenuates the vasodilator efficacy of protein kinase $\mathrm{G}$ in fetal and adult ovine cerebral arteries. American Journal of Physiology: Heart and Circulatory Physiology 313 H207-H219. (https:// doi.org/10.1152/ajpheart.00480.2016)

Williams JM \& Pearce WJ 2006 Age-dependent modulation of endothelium-dependent vasodilatation by chronic hypoxia in ovine cranial arteries. Journal of Applied Physiology 100 225-232. (https://doi. org/10.1152/japplphysiol.00221.2005)

Wu C, Li J, Bo L, Gao Q, Zhu Z, Li D, Li S, Sun M, Mao C \& Xu Z 2014 High-sucrose diets in pregnancy alter angiotensin II-mediated pressor response and microvessel tone via the PKC/Cav1.2 pathway in rat offspring. Hypertension Research 37 818-823. (https://doi.org/10.1038/ hr.2014.94)

Xiao D, Xu Z, Huang X, Longo LD, Yang S \& Zhang L 2008 Prenatal gender-related nicotine exposure increases blood pressure response to angiotensin II in adult offspring. Hypertension 51 1239-1247. (https:// doi.org/10.1161/HYPERTENSIONAHA.107.106203)

Xu Z, Wu Y, Zhang Y, Zhang H \& Shi L 2019 Melatonin activates BKCa channels in cerebral artery myocytes via both direct and MT receptor/ PKC-mediated pathway. European Journal of Pharmacology $\mathbf{8 4 2}$ 177-188. (https://doi.org/10.1016/j.ejphar.2018.10.032)

Zhang Y, Su W, Zhang Q, Xu J, Liu H, Luo J, Zhan L, Xia Z \& Lei S 2018 Glycine protects H9C2 cardiomyocytes from high glucoseand hypoxia/reoxygenation-induced injury via inhibiting PKCbeta2 activation and improving mitochondrial quality. Journal of Diabetes Research 20189502895 . (https://doi. org/10.1155/2018/9502895)

Zhao Y, Long W, Zhang L \& Longo LD 2003 Extracellular signal-regulated kinases and contractile responses in ovine adult and fetal cerebral arteries. Journal of Physiology 551 691-703. (https://doi.org/10.1113/ jphysiol.2003.046128)

Received in final form 14 October 2019

Accepted 23 October 2019

Accepted Manuscript published online 23 October 2019 https://joe.bioscientifica.com https://doi.org/10.1530/JOE-19-0370 (c) 2020 Society for Endocrinology Published by Bioscientifica Ltd. Printed in Great Britain 